Objectives It is widely accepted that HLA-G belongs to the group of checkpoint molecules, implicated in different pathways of immune suppression allowing tumors escape. Here, we investigated HLA-G expression in vulvar squamous cell carcinoma (VSCC) to explore the potential implication of these molecules as prognostic markers.

Methods Immunohistochemistry was performed to evaluate HLA-G expression using the monoclonal antibody anti-HLA-G clone $4 \mathrm{H} 84$ that specifically identifies the denatured heavy chain of all HLA-G isoforms. Association with clinicopathological factors and survival were analyzed in 56 VSCC treated with radical vulvectomy. Mann-Whitney (MW) U test was used to estimate differences in HLAG levels expression in subgroups. Survival estimation was calculated by the KaplanMeier test.

Results HLA-G was highly expressed in 11 of the 56 (19.6\%) primary tumor specimens. The high HLA-G expression level was reported in high-sized tumors $(\mathrm{PMW}=0.03)$ and increased invasion depth $(\mathrm{PMW}=0.01)$. HLA-G high expression was also noted in $72,7 \%$ of advanced stages with a borderline significance (PMW=0.08). A high level of HLA-G was not associated with tumors' resection margins $(\mathrm{PMW}=0.18)$. Assessment of patients' survival by Kaplan-Meier analysis indicated an adverse correlation between HLA-Ghigh expression and overall survival rate of VSCC patients (log-rank; $\mathrm{P}=0.000037$ ). Therefore, the 5-year cumulative survival rates of patients with HLA-Ghigh expression was $10.5 \%$. In the same way, HLA-G high expression reduced the disease-free survival $(\mathrm{P}=0.002)$.

Conclusions Our study shows that VSCC expresses high HLA$G$ that has been associated with an unfavorable clinical outcome. These findings suggest that HLA-G might be considered as a novel postoperative prognostic indicator for VSCC

\section{EPV286/\#444 TRENDS IN MORBIDITY OF VULVAR CANCER IN UZBEKISTAN}

S Djanklich*, Z Sabirdjanova. Republican Specialized Scientific-Practical Medical Center of Oncology and Radiology, Gynecological, Tashkent, Uzbekistan

\subsection{6/ijgc-2021-IGCS.357}

Objectives To analyze trends in cancer morbidity for vulvar cancer (VC) in Uzbekistan.

Methods We collected vulvar cancer incidence data from official statistics in Uzbekistan for the years $2017+2018$.

Results Totally 131 patients with VC have been registered, 61 in 2017 and 70 in 2018 of which rural women are 33, 40 and respectively. According to the age patients were registered as follows: in 2017 from 18 to 35 years old - 4 patients, from 36 to 55 years old - 14 patients, from 56 to 70 years old - 35 patients, older than 70 years old - 19 patients;in 2018 from the age of 18 up to 35 years old 1 patient, 36-55 years old - 20 patients, 56-70 years old - 24 patients and 16 patients are older than 70 years. In 2017, the largest number of VC patients ware registered in Tashkent (17), Kashkadarya (10), Bukhara (10) and Tashkent regions (8). In 2018, there was an increase in the number of VC patients in the Republic of Karakalpakstan (from 4 to 13), in the Khorezm region (from 5 to 9), while in Tashkent and the Tashkent region the number of registered cases of VC decreased ( 9 and 3, respectively). In 2018, the number of VC cases increased in
Tashkent region, Tashkent and Namangan (10, 9 and 9 respectively). In Syrdarya, Jizzakh and Navoi regions in 2017-18 years there were no cases of VC.

Conclusions Screening programs will allow timely diagnosis of vulvar background and precancerous diseases and reduce the number of patients with this pathology.

\section{EPV287/\#539 SURGICAL TREATMENT OF VULVAR CARCINOMA IN VERY ELDERLY ITALIAN POPULATION: A RETROSPECTIVE STUDY}

${ }^{1} \mathrm{G}$ Falcicchio*, ${ }^{2} \mathrm{~V}$ Loizzi, ${ }^{1} \mathrm{E}$ Cicinelli, ${ }^{1} \mathrm{~F}$ Arezzo, ${ }^{3} \mathrm{~L}$ Resta, ${ }^{3} \mathrm{G}$ Serio, ${ }^{3} \mathrm{G}$ Cazzato, ${ }^{1} \mathrm{~A}$ Esposito. 'University of Bari 'Aldo Moro', Department of Biomedical Science and Human Oncology, Obstetrics and Gynecology Unit, Bari, Italy; 'University of Bari 'Aldo Moro', Interdisciplinar Department of Medicine, Obstetrics and Gynecology Unit, Bari, Italy; ${ }^{3}$ University of Bari 'Aldo Moro', Emergency and Organ Transplantation, Pathology Section, Bari, Italy

\subsection{6/ijgc-2021-IGCS.358}

Objectives Vulvar carcinoma (VC) is a rare condition: there is a lack of evidence on treatment in Very Elderly (VE) patients. Aim of the study is to evaluate outcome of surgical resection of VC in the VE population (80 or more years).

Methods Age at diagnosis, FIGO stage, surgical management, groin involvement, site of relapse, disease free survival and overall survival (OAS) were collected for each patient.

Results 32 patients were managed between 2000 and 2020. Mean age at diagnosis was 82.8 years [80-92 years]. Surgical treatment consisted of radical vulvectomy in $22(68.7 \%)$ cases and wide local excision in $10(31.3 \%)$. All patients underwent groin node dissection: 17 (53.2\%) monolateral, 15 (46.8\%) bilateral. FIGO stage was I in 18 cases (56.3\%), II in 1 case (3.1\%), 12 had positive nodes (stage III) (37.5\%) and 1 case had stage IV (3.1\%). Adjuvant radiotherapy was delivered in 5 patients (15.6\%). Most common complication was wound breakdown that occurred in 2 cases (6.3\%). 15 patients (46.9\%) were lost at follow-up. 5 vulvar and groin recurrences were observed (29.4\%). Median time to relapse was 21 months [5-47]. Mean OAS was 59.9 months for patients with negative nodes and 13 months for those with positive nodes. Overall survival for the entire group was 35 months [2-148] and $7(21.9 \%)$ patients are alive without evidence of disease. Conclusions Surgical management of VC is feasible even in the VE population. Complication rate is acceptable, groin dissection appears to have a prognostic rather than a therapeutic purpose.

\section{EPV288/\#61 VERRUCOUS CARCINOMA OF THE VULVA: PATTERNS OF CARE AND TREATMENT OUTCOMES}

${ }^{1} S$ Dryden, ${ }^{2} \mathrm{~J}$ Gaskins, ${ }^{3} \mathrm{~L}$ Reshko, ${ }^{3} \mathrm{~A}$ Rattani, ${ }^{3} \mathrm{~S}$ Silva*. ${ }^{1}$ University of Louisville, Gynecologic Oncology, Louisville, USA; ${ }^{2}$ University of Louisville, Bioinformatics and Biostatistics, Louisville, USA; ${ }^{3}$ University of Louisville, Radiation Oncology, Louisville, USA

\subsection{6/ijgc-2021-IGCS.359}

Objectives Verrucous vulvar carcinoma (VC) is an uncommon and distinct histologic subtype of squamous cell carcinoma (SCC). The goals of this study were to analyze patient data from the National Cancer Database (NCDB) to quantitate the incidence of $\mathrm{VC}$ and to investigate the effects of patient and tumor demographic factors and treatment regimens on overall survival (OS). 